Supporting Information

\title{
Graphene Wrinkles Enable Spatially Defined Chemistry
}

Shikai Deng ${ }^{1}$, Dongjoon Rhee ${ }^{2}$, Won-Kyu Lee ${ }^{2}$, Songwei Che ${ }^{3}$, Bijentimala Keisham ${ }^{3}$, Vikas Berry $^{3}$ and Teri W. Odom ${ }^{1,2}$ *

${ }^{1}$ Department of Chemistry, and ${ }^{2}$ Department of Materials Science and Engineering, Northwestern University, Evanston, Illinois 60208, United States, and ${ }^{3}$ Department of Chemical Engineering, University of Illinois at Chicago, Chicago, Illinois 60607, United States

Corresponding author: todom@northwestern.edu

METHODS S3

Conformal contact between graphene and skin layer .......................................................... S7

Fluorination of 2D graphene wrinkles formed with different strains...................................S8

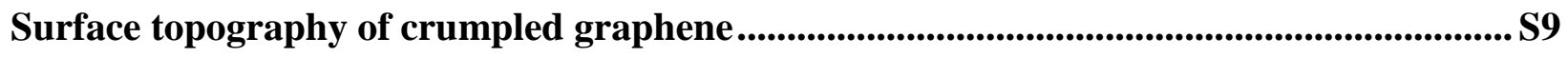

Raman spectra of PS and skin layer with and without graphene before fluorination ........S10

Raman spectra of skin layer before and after fluorination....................................................S11

Raman spectra of graphene crumples before and after fluorination................................S12

SEM images of graphene wrinkles and crumples ......................................................................S13

AFM of graphene patterns with wrinkles and crumples ......................................................... S14

AFM of graphene wrinkles and crumples formed with different domain widths ..............S15

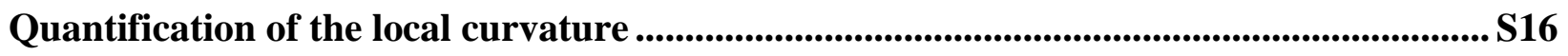

Local curvature at peaks of graphene wrinkles and crumples..............................................S17

Mapping of Raman $I_{2 D} / I_{G}$ ratios on graphene wrinkles and crumples side-by-side........... S18

Raman mapping of fluorinated graphene with different pattern geometries ......................S19 
Current mapping of patterned graphene wrinkles and crumples before fluorination ...... S20

Contact between tip and surface in conductive AFM mode ...........................................S21

Multiscale wrinkles and crumples in double-skin patterned graphene .................................. S22

Current mapping of multiscale wrinkles and crumples in graphene before fluorination .S23

Local current of double-skin patterned graphene after reaction ......................................S24

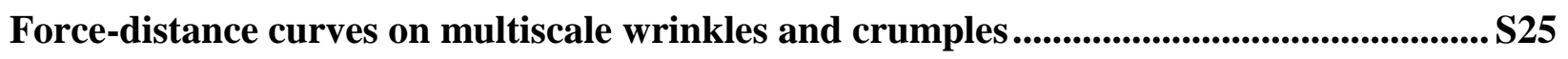




\section{METHODS}

Fluoropolymer skin layer formation. Pre-strained polystyrene (PS) films (Shrinky Dinks, Grafix) were rinsed with isopropyl alcohol (IPA) and distilled water, followed by drying with air. The PS substrates were then treated with $\mathrm{CHF}_{3}$ plasma in a reactive ion etching (RIE) system for 20 seconds at a flow rate of $25 \mathrm{sccm}$ under $20 \mathrm{~Pa}$ at a power of $70 \mathrm{~W}$. The fluoropolymer skin layer formed on the surface at a rate of $\sim 0.5 \mathrm{~nm} / \mathrm{s}$.

Graphene synthesis and transfer. Pretreated $\mathrm{Cu}$ foils $(99.8 \%$, annealed, uncoated, Alfa-Aesar) were placed inside a home-built low-pressure chemical vapor deposition (LP-CVD) system with a standard 1-inch quartz tube reactor. The reaction chamber was first evacuated to $\sim 1.5$ mTorr and then flushed with $\mathrm{H}_{2}$ at a flow rate of $100 \mathrm{sccm}$ (99.9999\% purity, Praxair) for 20 minutes under a total pressure of $650 \mathrm{mTorr}$. The temperature was then increased to $1050^{\circ} \mathrm{C}$ in 25 minutes $(\sim 40$ ${ }^{\circ} \mathrm{C} / \mathrm{min}$ ) under a hydrogen flow at $10 \mathrm{sccm}$. The $\mathrm{Cu}$ foil was annealed at this temperature to increase the grain size and to smoothen the surface. Subsequently, monolayer graphene was synthesized by introducing $\mathrm{CH}_{4}$ (99.999\% purity, Praxair) and $\mathrm{H}_{2}$ gases at flow rates of $10 \mathrm{sccm}$ (partial pressure: 10 mTorr) and $22 \mathrm{sccm}$ (partial pressure: $\sim 270$ mTorr) for 20 minutes. After the graphene growth, the reaction chamber was cooled down to room temperature in 40 minutes $\left(\sim 25^{\circ} \mathrm{C} / \mathrm{min}\right)$.

Graphene was transferred onto PS substrates using poly(methyl-methacrylate) (PMMA) as a support layer. After spin coating PMMA solution (PMMA 950 C, MicroChem) on one side of the $\mathrm{Cu}$ foil, we removed graphene on the back side with $\mathrm{O}_{2} \mathrm{RIE}$ at a flow rate of $15 \mathrm{sccm}$, a pressure of $1.3 \mathrm{~Pa}$, and a power of $10 \mathrm{~W}$ for one minute. Copper was then etched using a dilute $(\mathrm{NH} 4)_{2} \mathrm{~S}_{2} \mathrm{O}_{8}$ solution $(60 \mathrm{mM})$ for 4 hours. The resulting PMMA/graphene bilayer was floated on 
three consecutive DI water baths (20 minutes each) to remove residual ions or impurities, and then transferred onto the PS substrate. The PMMA/graphene/PS sample was dried overnight and subsequently placed in an acetic acid bath for 10 mins to remove PMMA. The residual acetic acid and PMMA was rinsed by ethanol.

Fabrication of graphene wrinkles and crumples. After graphene transfer, $\mathrm{CHF}_{3}$-treated PS sheets were heated in an oven at $130{ }^{\circ} \mathrm{C}$ to relieve pre-strain in the substrate and form graphene wrinkles. Graphene crumples were produced by the same process with PS substrates without skin layers. For 1D wrinkles and crumples, strain $\left(\varepsilon_{1 \mathrm{D}}\right)$ was determined based on the change in length of the PS substrate before and after the strain relief: $\varepsilon_{1 \mathrm{D}}=\left(L_{0}-L_{\mathrm{R}}\right) / L_{0}$, where $L_{0}$ and $L_{\mathrm{R}}$ are the lengths before and after shrinking. For 2D samples, we calculated strain $\left(\varepsilon_{2} \mathrm{D}\right)$ from the areal change of the PS substrate before and after strain relief using the equation $\varepsilon_{2 \mathrm{D}}=\left(A_{0}-A_{\mathrm{R}}\right) / A_{0}$, in which $A_{0}$ and $A_{\mathrm{R}}$ are the initial and final areas.

Formation of wrinkles and crumples side-by-side. To form patterned skin layers, the PS substrate was first covered with polyvinylpyrrolidone (PVP) lines. First, PVP/ethanol solution was dropped on the PS substrate and then molded with a poly(dimethylsiloxane) (PDMS) mask having recessed line patterns. Parallel PVP line features were formed after evaporating ethanol overnight and detaching the PDMS. Treating the masked PS substrate with $\mathrm{CHF}_{3} \mathrm{RIE}$ formed skin layers on the exposed PS regions. PVP was then removed by sonicating the sample in ethanol for $1 \mathrm{~min}$, which resulted in the line-patterned of skin layers on the PS surface. Relieving pre-strain in the patterned substrate after graphene transfer formed wrinkles (on skin) and crumples (on PS) side- 
by-side. For multiscale graphene patterns with four different feature sizes, two cycles of the skinpatterning process were performed on PS with lines aligned perpendicular to each other, followed by graphene transfer and strain relief. The advantage of our strategy is that mask residue on graphene can be minimized because the skin layer patterning is performed prior to the graphene transfer. Additionally, the single-step reaction approach can also avoid potential damages in graphene involved with conventional area-selective functionalization methods based on multiplestep mask protection and reaction.

CF4 plasma reaction. Graphene was fluorinated by a $\mathrm{CF}_{4}$ plasma treatment in a $\mathrm{RIE}$ chamber with the following processing conditions: a flow rate of $25 \mathrm{sccm}$, a pressure of $200 \mathrm{mTorr}$ and a power of $10 \mathrm{~W}$ for 5 seconds.

Raman spectroscopy. The Raman spectroscopic data were obtained using WITEC Raman Alpha 300-RA with a laser excitation wavelength of $532 \mathrm{~nm}$. All the samples were probed using an 100X objective. The spot size was about $700 \mathrm{~nm}$. Raman mapping was performed on $10 \times 10 \mu \mathrm{m}^{2}$ areas with 40 to 50 spots per $10 \mu \mathrm{m}$ (step size $<300 \mathrm{~nm}$ ). The average and standard deviation in Figure 2 were determined based on these 1600-2500 data points.

Quantification of local curvature. The AFM distance (x)-height (z) data points from wrinkle/crumple peaks were fitted by a third-degree polynomial function. The local curvature was calculated using the formal definition $\left.\kappa=\left|z^{\prime \prime}\right| /\left[1+\left(z^{\prime}\right)^{2}\right]\right)^{3 / 2}$, where $z^{\prime}$ and $z^{\prime \prime}$ are the first and second derivatives of $z$ with respect to $x$. The average peak curvatures of graphene wrinkles and crumples were determined from 20 measurements. 
Conductive AFM characterization. For conductive AFM measurements, an electrode was formed on multiscale graphene wrinkles and crumples by depositing 50-nm osmium through a glass slide that partially masked the surface. The measurement was conducted using a doped silicon cantilever coated with platinum and iridium in contact mode.

AFM indentation characterization. The force-distance curves on different regions of wrinkles and crumples before and after reaction were obtained using AFM tips with a spring constant of $38.1 \mathrm{nN} / \mathrm{nm}$. Stiffness and adhesion force were determined from 10 to 15 measurements on different areas. 
Conformal contact between graphene and skin layer

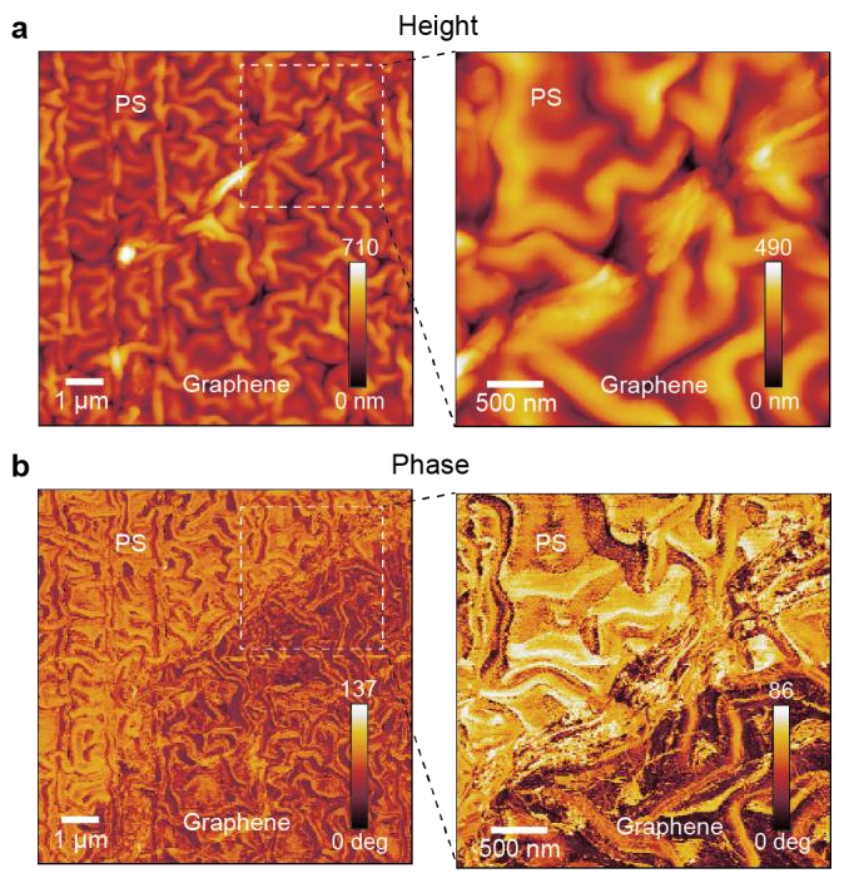

Figure S1. Conformal contact between graphene and skin layer. (a) Height and (b) phase images near the boundary between graphene and PS regions. 


\section{Fluorination of 2D graphene wrinkles formed with different strains}

a
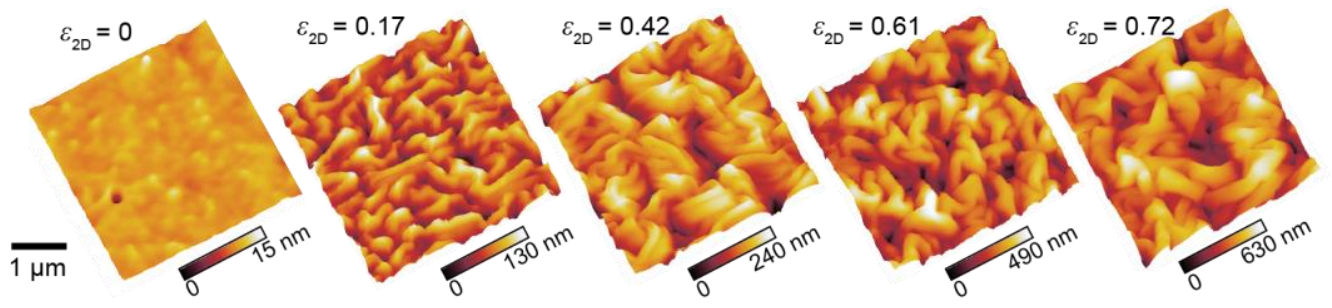

b
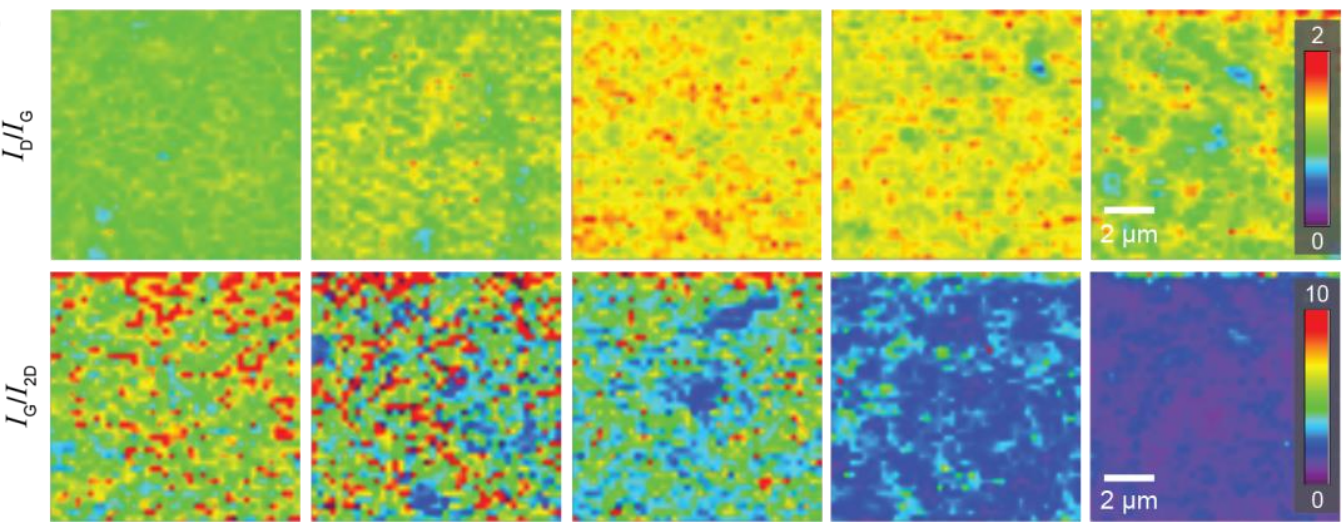

Figure S2. Fluorination of 2D graphene wrinkles formed with different strains. (a) AFM height images of 2D graphene wrinkles formed after different amounts of strain relief. (b) Mapping of Raman $I_{\mathrm{D}} / I_{\mathrm{G}}$ (top row) and $I_{\mathrm{G}} / I_{2 \mathrm{D}}$ (bottom row) ratios of the graphene wrinkles. 


\section{Surface topography of crumpled graphene}
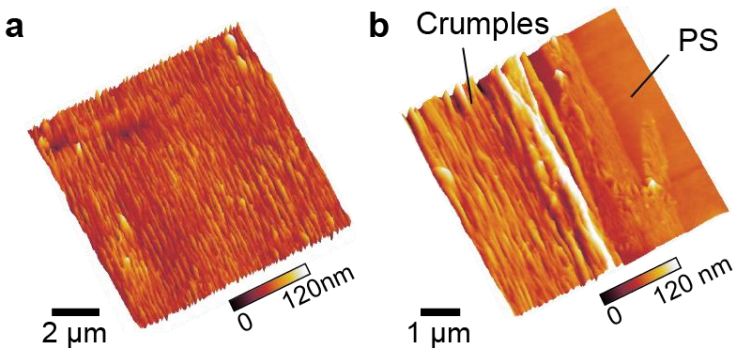

C

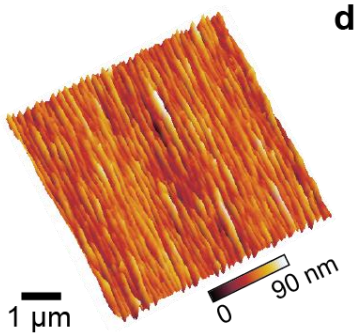

d

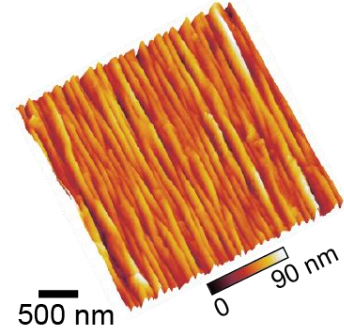

Figure S3. Surface topography of crumpled graphene. (a) Three-dimensional AFM height images of crumples over $10 \times 10 \mu \mathrm{m}^{2}$ area. (b) Height image of crumpled graphene near the boundary between graphene and PS. Three-dimensional height image of crumples over (c) $6 \times 6 \mu \mathrm{m}^{2}$ and (d) $3 \times 3 \mu \mathrm{m}^{2}$ areas. 


\section{Raman spectra of PS and skin layer with and without graphene before fluorination}

a

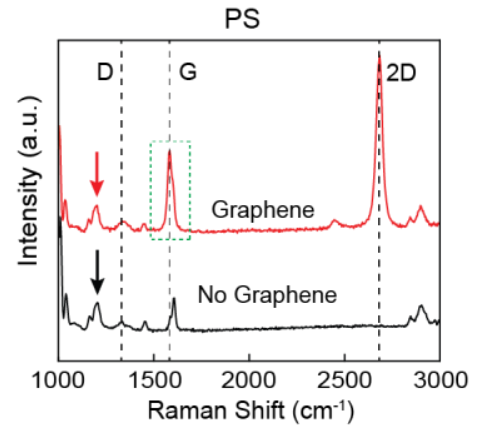

c

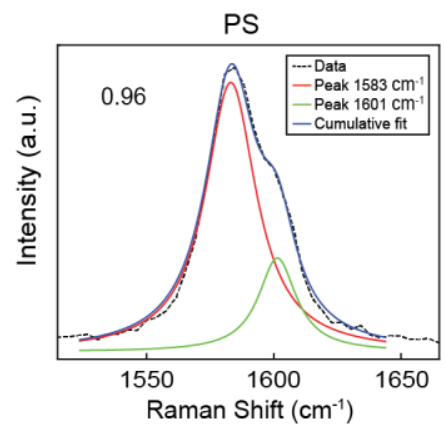

b

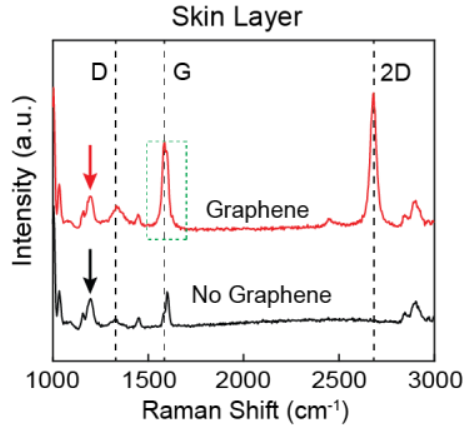

d

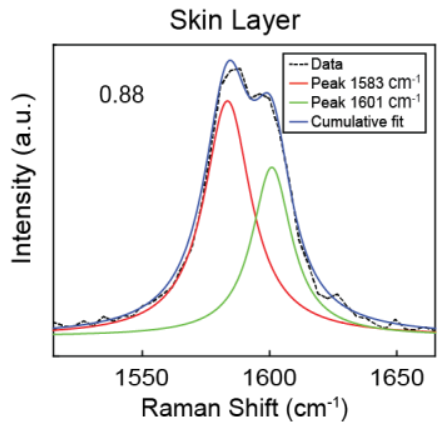

Figure S4. Raman spectra of PS and skin layer with and without graphene before fluorination. (a) Raman spectra of bare PS substrate and graphene on PS. (b) Raman spectra of the flat skin layer on PS and graphene on the skin layer. The G peak of graphene at 1583 $\mathrm{cm}^{-1}$ (red line) partially overlapped with the background peak at $1601 \mathrm{~cm}^{-1}$ (black line). The reference peak at $1196 \mathrm{~cm}^{-1}$ is highlighted with arrows in (a) and (b). (c) Deconvolution of the broadened $\mathrm{G}$ peak in dashed green rectangle of (a). The ratio of fitted $\mathrm{G}$ peak intensity (red line) to that of broadened G peak (black dashed line) on bare PS was 0.96. (d) Deconvolution of the broadened $\mathrm{G}$ peak in dashed green rectangle of (b). The ratio of fitted $\mathrm{G}$ peak intensity (red line) to that of original data (black dashed line) on skin layer was 0.88 . 


\section{Raman spectra of skin layer before and after fluorination}

a

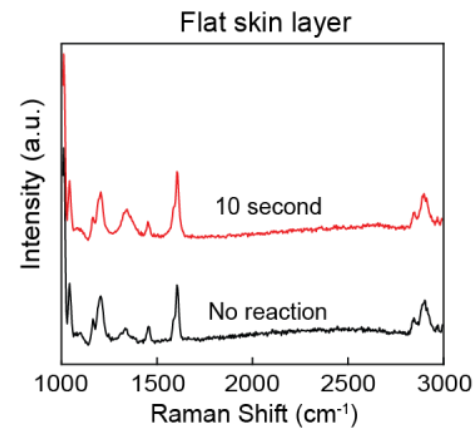

b

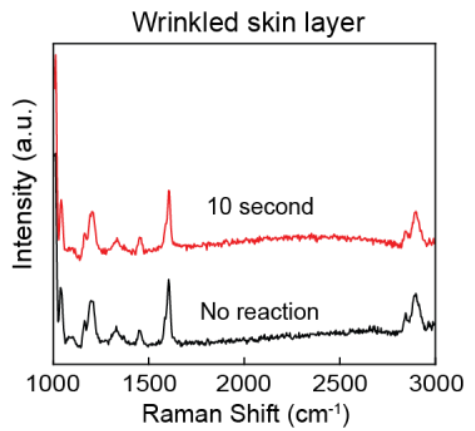

Figure S5. Raman spectra of skin layer before and after fluorination. Raman spectra of (a) flat and (b) wrinkled skin layers before and after fluorination. $\mathrm{The} \mathrm{CF}_{4}$ plasma treatment time for both samples was 10 seconds. 
Raman spectra of graphene crumples before and after fluorination

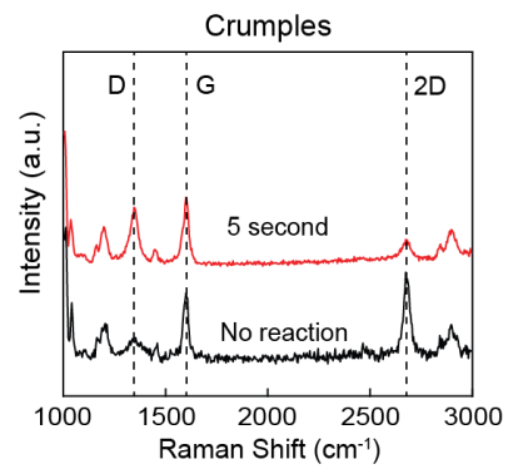

Figure S6. Raman spectra of graphene crumples before and after fluorination. $\mathrm{The} \mathrm{CF}_{4}$ plasma treatment time was 5 seconds. 
SEM images of graphene wrinkles and crumples
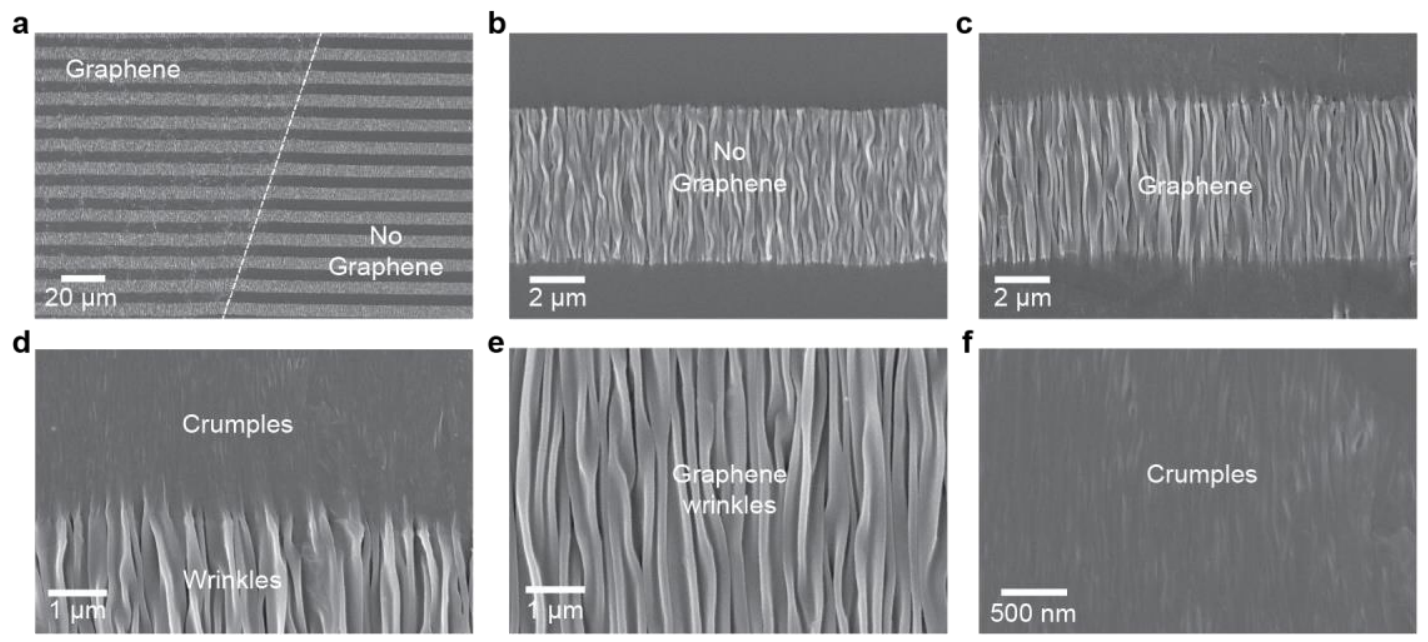

Figure S7. SEM characterization of graphene wrinkles and crumples side-by-side. (a) Boundary between graphene and bare PS regions. (b) Skin layer wrinkles without graphene and (c) graphene wrinkles on the skin layer. (d) Boundary between wrinkles and crumples. (e) Conformally wrinkled graphene on the skin layer. (f) Crumpled regions on bare PS. 
AFM of graphene patterns with wrinkles and crumples
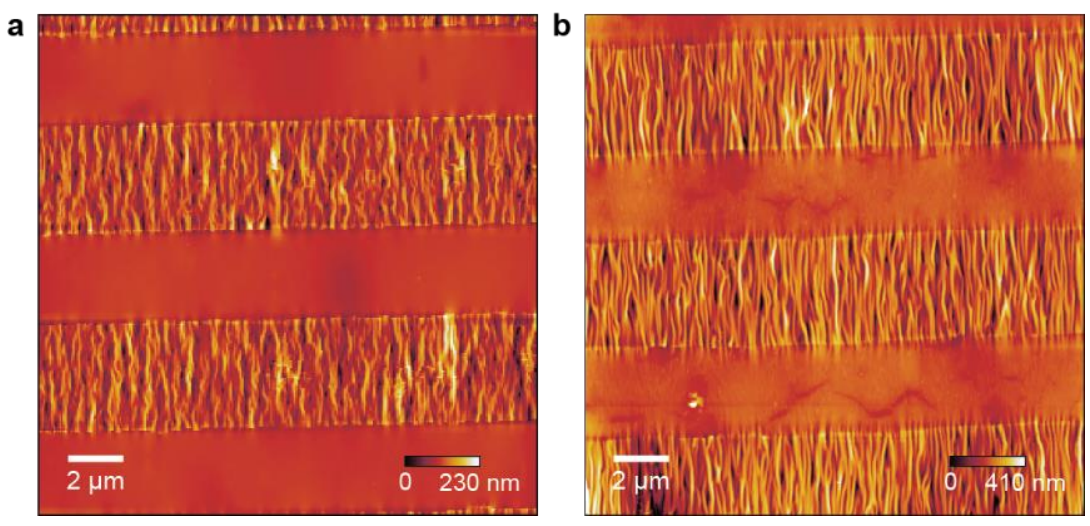

Figure S8. AFM characterization of PS and graphene wrinkles. (a) 1D wrinkles in skin layer without graphene and (b) graphene wrinkles and crumples side-by-side. The width of line patterns was $5 \mu \mathrm{m}$. 
AFM of graphene wrinkles and crumples formed with different domain widths
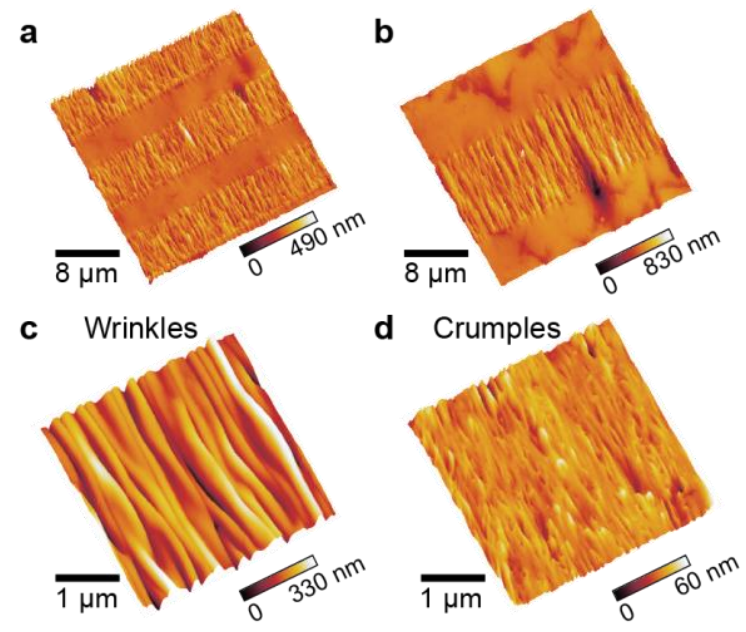

Figure S9. Surface topography of wrinkles and crumples. AFM height images of side-byside graphene wrinkles and crumples formed with different line widths: (a) $5 \mu \mathrm{m}$ and (b) 10 $\mu \mathrm{m}$. Images of (c) wrinkles and (d) crumples at a higher magnification. 


\section{Quantification of the local curvature}
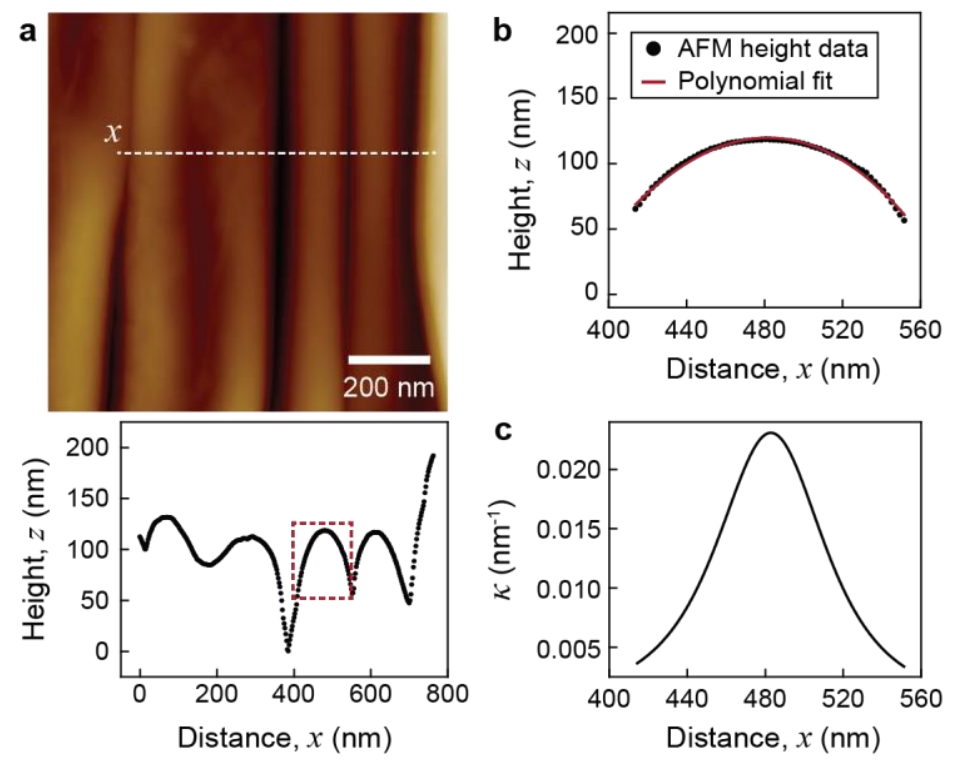

Figure S10. Calculation of the local curvature from AFM height data. (a) Representative AFM image and height profile of graphene wrinkles. (b) Polynomial fitting to AFM data points from a wrinkle peak (marked with a red box in (a)). (c) Calculated local curvature. 
Local curvature at peaks of graphene wrinkles and crumples

Table S1. Calculated local curvature at peaks of graphene wrinkles and crumples

\begin{tabular}{|c|c|c|}
\hline \multirow{2}{*}{ Measurements } & \multicolumn{2}{|c|}{ Peak curvature $\left(\mathbf{n m}^{-\mathbf{1}}\right)$} \\
\cline { 2 - 3 } & Wrinkles & Crumples \\
\hline 1 & 0.0193 & 0.0869 \\
\hline 2 & 0.0211 & 0.0365 \\
\hline 3 & 0.0206 & 0.0831 \\
\hline 4 & 0.0195 & 0.0641 \\
\hline 5 & 0.0253 & 0.0436 \\
\hline 6 & 0.0212 & 0.0470 \\
\hline 7 & 0.0186 & 0.0990 \\
\hline 8 & 0.0230 & 0.0454 \\
\hline 9 & 0.0183 & 0.0446 \\
\hline 10 & 0.0202 & 0.0402 \\
\hline 11 & 0.0238 & 0.0470 \\
\hline 12 & 0.0205 & 0.0392 \\
\hline 13 & 0.0247 & 0.0415 \\
\hline 14 & 0.0206 & 0.0343 \\
\hline 15 & 0.0244 & 0.0472 \\
\hline 16 & 0.0207 & 0.0521 \\
\hline 17 & 0.0259 & 0.0491 \\
\hline 18 & 0.0261 & 0.0556 \\
\hline 19 & 0.0168 & 0.0429 \\
\hline 20 & 0.0234 & 0.0552 \\
\hline Average & $\mathbf{0 . 0 2 1 7}$ & $\mathbf{0 . 0 5 2 7}$ \\
\hline Standard deviation & $\mathbf{0 . 0 0 2 7 0}$ & $\mathbf{0 . 0 1 7 6}$ \\
\hline & & \\
\hline
\end{tabular}


Mapping of Raman $I_{2 D} / I_{G}$ ratios on graphene wrinkles and crumples side-by-side
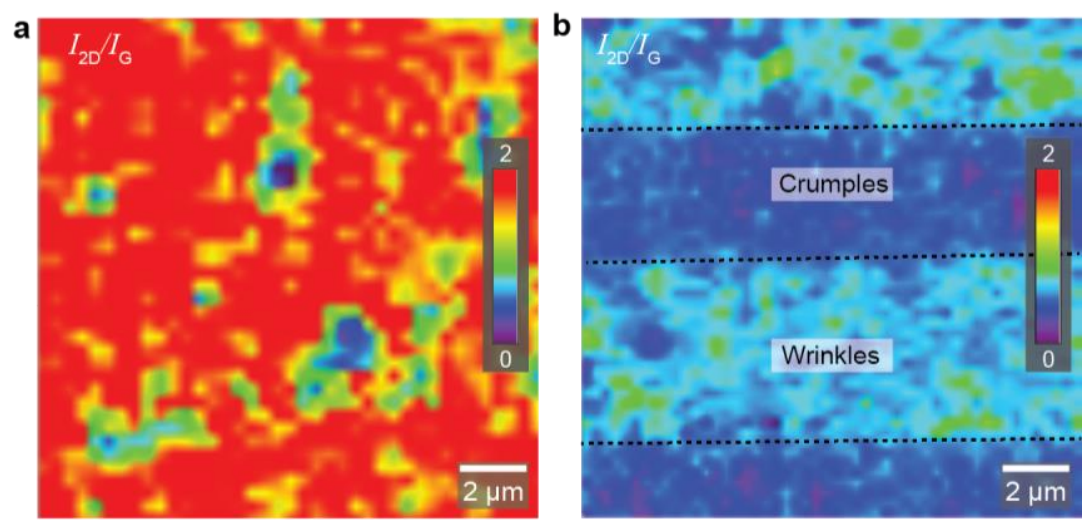

Figure S11. Raman $I_{2 D} / I_{G}$ ratio mapping of graphene wrinkles and crumples before and after fluorination. (a) $I_{2 D} / I_{G}$ ratio mapping before reaction. The values were from 1.7 to 2 in most areas. (b) $I_{2 D} / I_{G}$ ratio mapping after reaction. Crumpled regions showed smaller values than wrinkled areas. 


\section{Raman mapping of fluorinated graphene with different pattern geometries}
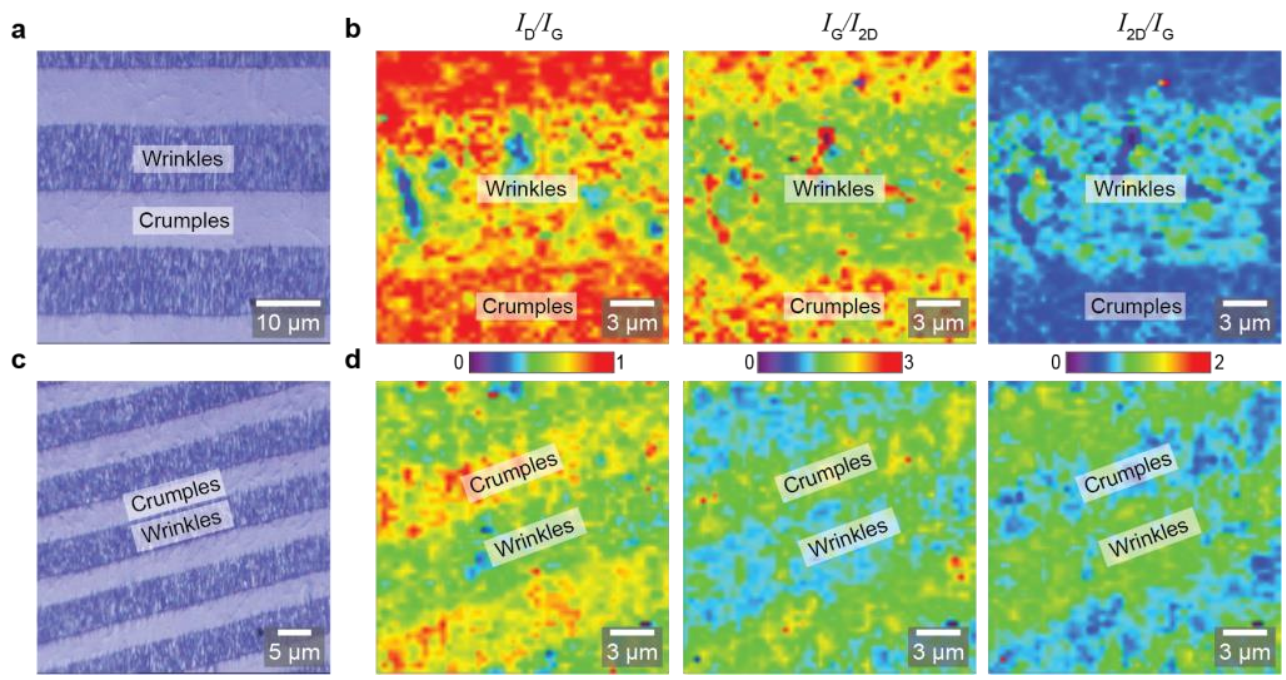

Figure S12. Fluorination of graphene wrinkles and crumples with different line widths and feature orientations. (a) Optical image and (b) Raman $I_{D} / I_{G}, I_{G} / I_{2 D}$, and $I_{2 D} / I_{G}$ mapping of graphene wrinkles and crumples with a line width of $10 \mu \mathrm{m}$ after fluorination. (c) Optical image and (d) Raman $I_{D} / I_{G}, I_{G} / I_{2 D}$, and $I_{2 D} / I_{G}$ mapping of graphene wrinkles and crumples with a line width of $5 \mu \mathrm{m}$ after fluorination. The angle between patterned skin and 1D strain direction was $\sim 15^{\circ}$. 
Current mapping of patterned grapheme wrinkles and crumples before fluorination

a

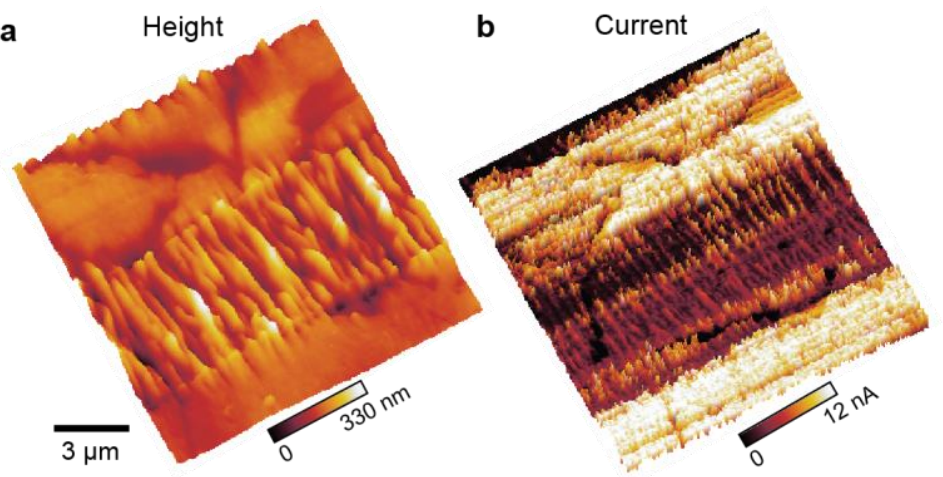

Figure S13. Conductive AFM characterization of single-skin patterned grapheme wrinkles and crumples before fluorination. (a) Height and (b) current mapping.

S20 
Contact between tip and surface in conductive AFM mode

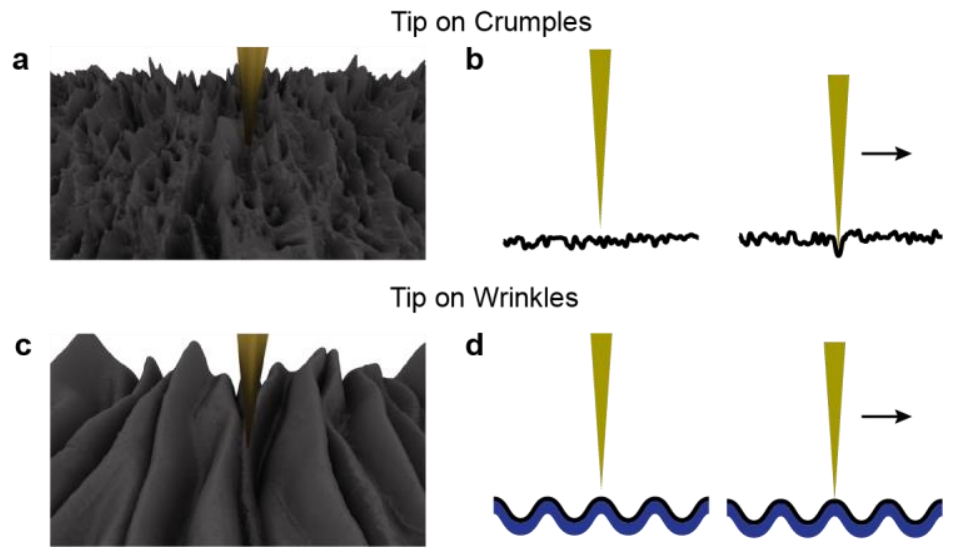

Figure S14. Scheme illustrating the contact between tip and surface on graphene wrinkles and crumples. (a) Tip-crumple contact. (b) Cross-sectional view of tip and crumples before contact (left) and during scan (right). (c) Tip-wrinkle contact. (d) Crosssectional view of tip before contact (left) and during scan (right). 
Multiscale wrinkles and crumples in double-skin patterned graphene
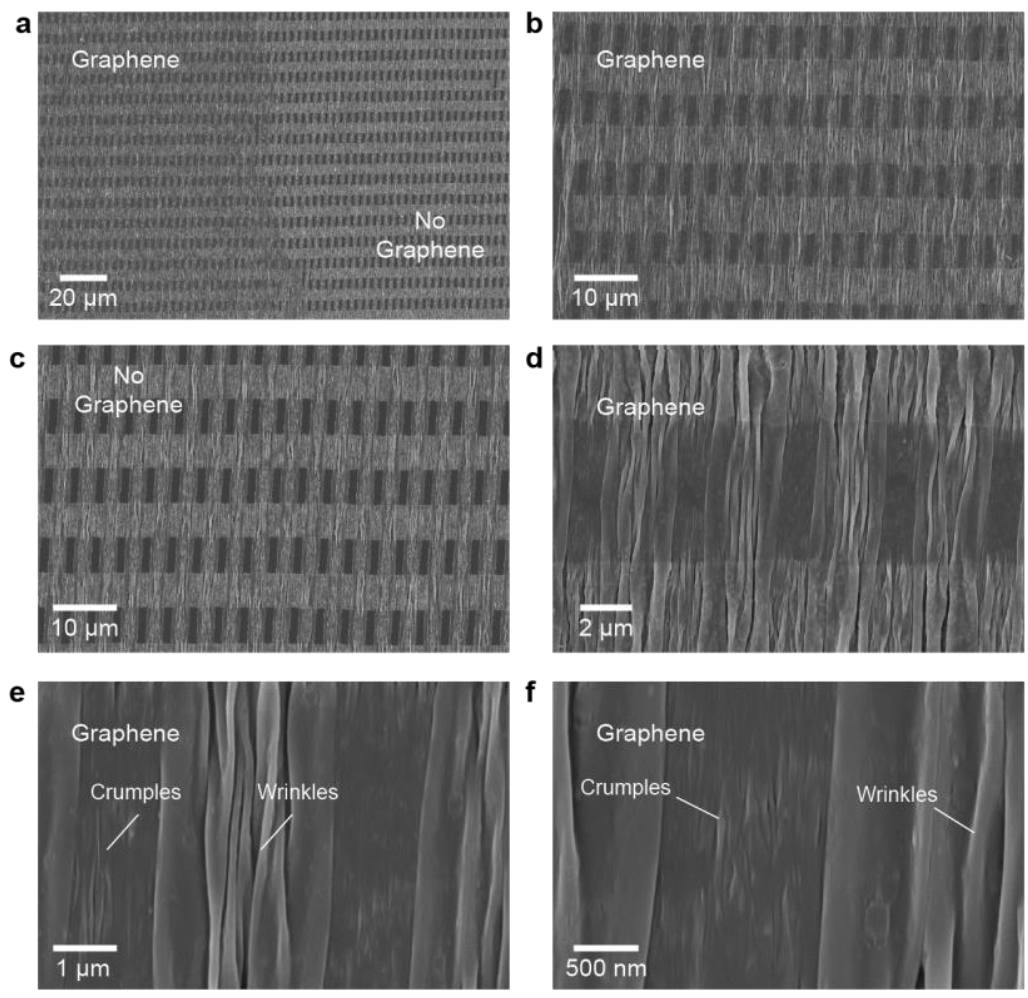

Figure S15. SEM images of multiscale wrinkles and crumples on double-skin coated PS. (a) Boundary between graphene and uncoated regions after strain relief. Multiscale features on (b) graphene and (c) skin layer without graphene. (d) to (f) Zoomed-in images of graphene wrinkles and crumples. 
Current mapping of multiscale wrinkles and crumples in graphene before fluorination

a
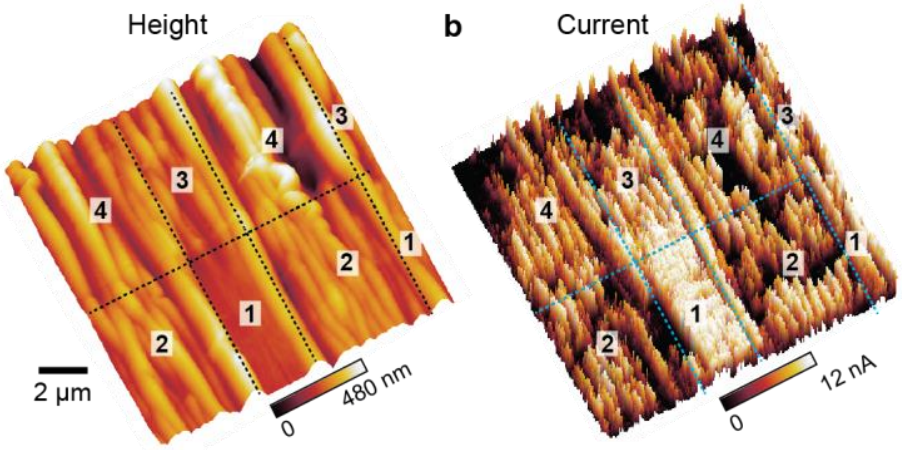

Figure S16. Conductive AFM characterization of multiscale wrinkles and crumples in graphene before fluorination. (a) Height and (b) current mapping of double-skin patterned graphene. 
Local current of double-skin patterned graphene after reaction
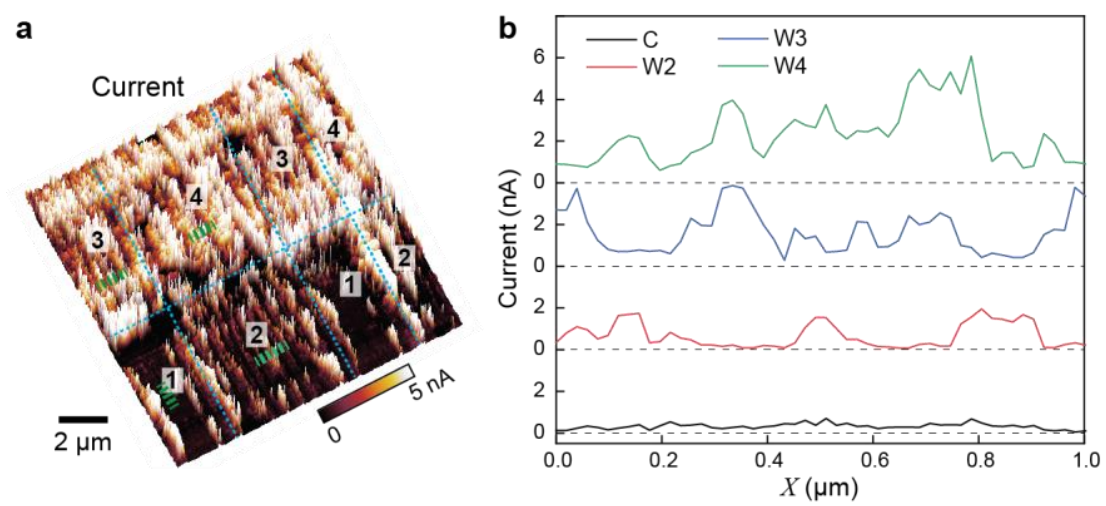

Figure S17. Local currents in different regions of patterned graphene after fluorination. (a) Current mapping of double-skin patterned graphene in a $10 \times 10 \mu \mathrm{m}^{2}$ area. (b) Current profiles along green dashed lines in (a). 
Force-distance curves on multiscale wrinkles and crumples
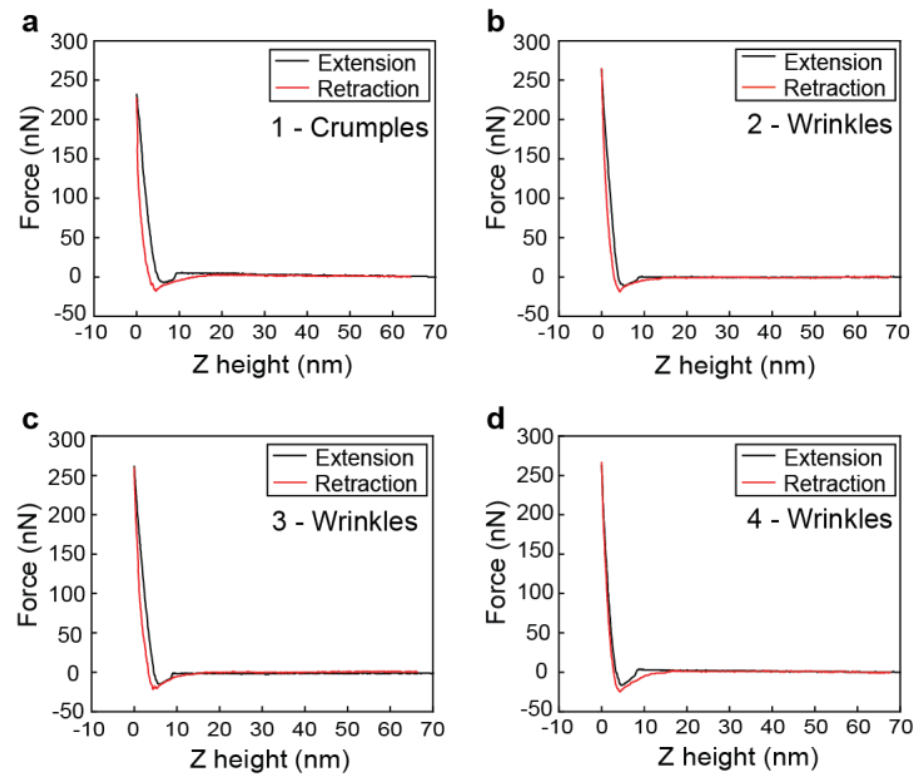

Figure S18. Tip indentation measurements on multiscale wrinkles and crumples. Typical force-distance curves of (a) crumples, (b) wrinkles in region 2, (c) wrinkles in region 3, and (d) wrinkles in region 4. 